\title{
CALET perspectives for calorimetric measurements of high energy electrons based on beam test results
}

\author{
Gabriele Bigongiari* \\ University of Siena \\ E-mail: bigongiari2@unisi.it
}

\begin{abstract}
CALET is a space mission currently in the final phase of preparation for a launch to the International Space Station (ISS), where it will be installed on the Exposure Facility of the Japanese Experiment Module (JEM-EF). One of the main science goals of the experiment is the measurement of the inclusive electron (+positron) spectrum. By integrating a sufficient exposure on the ISS, CALET will be able to explore the energy region above $1 \mathrm{TeV}$, where the presence of nearby sources of acceleration is expected to shape the high end of the electron spectrum and leave faint, but detectable, footprints in the anisotropy. In order to meet this experimental goal, CALET has been designed to achieve a large proton rejection capability $\left(>10^{5}\right)$ thanks to a full containment of electromagnetic showers in a $27 X_{0}$ thick calorimeter (TASC) preceded by a $3 X_{0}$ fine-grained pre-shower calorimeter (IMC) with imaging capabilities. In this paper the expected performance of the instrument with electrons will be discussed on the basis of the results of measurements performed during beam calibration tests at CERN-SPS at beam energies up to $290 \mathrm{GeV}$.
\end{abstract}

The 34th International Cosmic Ray Conference,

30 July- 6 August, 2015

The Hague, The Netherlands

* On behalf of the CALET collaboration 


\section{Introduction}

CALET (CALorimeteric Electron Telescope) is a Japanese led international mission funded by the Japanese Space Agency (JAXA), the Italian Space Agency (ASI), and NASA. The CALET instrument will be launched by a Japanese carrier and attached to the Exposure Facility (JEM-EF) on the International Space Station (ISS). The mission is expected to begin operations on the ISS in 2015 for a mission lifetime of 5 years.

The primary science goal of CALET is to perform high precision measurements of the electron spectrum from $1 \mathrm{GeV}$ to $20 \mathrm{TeV}$. By integrating a sufficient exposure, CALET will be able to detect the possible presence of nearby sources of acceleration by studying the shape of the electron spectrum above $1 \mathrm{TeV}$. CALET will produce its accurate scan of the electron and gamma ray spectra by utilizing its high energy resolution and a low background contamination provided by the excellent proton rejection capability of the instrument. This is made possible by a full containment of the e.m. showers in the calorimeter at these energies and a detailed imaging of the first 3 radiation length.

The precise measurement of the line shape of any spectral feature is expected to play a crucial role in the discrimination among different models of dark matter candidates, or it might suggest an alternative astrophysical interpretation.

CALET will also monitor gamma ray transients with a dedicated Gamma-ray Burst Monitor (CGBM) and study solar modulation.

Equipped with a charge identifier module, placed at the top of the apparatus, and capable to identify the atomic number $\mathrm{Z}$ of the incoming cosmic ray, CALET will perform long exposure observations of cosmic nuclei from proton to iron and will detect trans-iron elements with a dynamic range up to $Z=40$. It will extend, by one order of magnitude in energy, the present measurements of the ratio of secondary to primary elements (e.g.: boron/carbon and sub-iron/iron), thereby providing information about the energy dependence of cosmic-ray propagation in the galaxy.

In this paper, we report on a dedicated beam test measurement that took place at CERN in 2012 to assess the performance of the calorimeter system with electrons at beam energies up to $290 \mathrm{GeV}$ using an engineering model with similar readout and sensors to CALET Flight Model.

\section{CALET instrument}

CALET is an all-calorimetric instrument, with a total thickness of 30 radiation length $\left(\mathrm{X}_{0}\right)$ and 1.3 proton interaction length $\left(\lambda_{I}\right)$, preceded by a particle identification system. The energy measurement relies on two kinds of calorimeters: a fine grained pre-shower, known as imaging calorimeter (IMC), followed by a total absorption calorimeter (TASC). The effective geometrical factor of CALET for high energy electrons is $\simeq 1200 \mathrm{~cm}^{2}$ sr. The total weight of the system will be approximately $650 \mathrm{~kg}$.

The schematic structure of the whole instrument with the main sub-detectors can be seen in Fig. 1. The Gamma-ray Burst Monitor is described elsewhere [1].

In order to identify individual chemical elements in the cosmic-ray flux, a Charge Detector (CHD) is positioned at the top of the CALET instrument to provide a measurement of the electric charge of the incoming particle via the $Z^{2}$ dependence of the specific ionization loss in a double layered, 


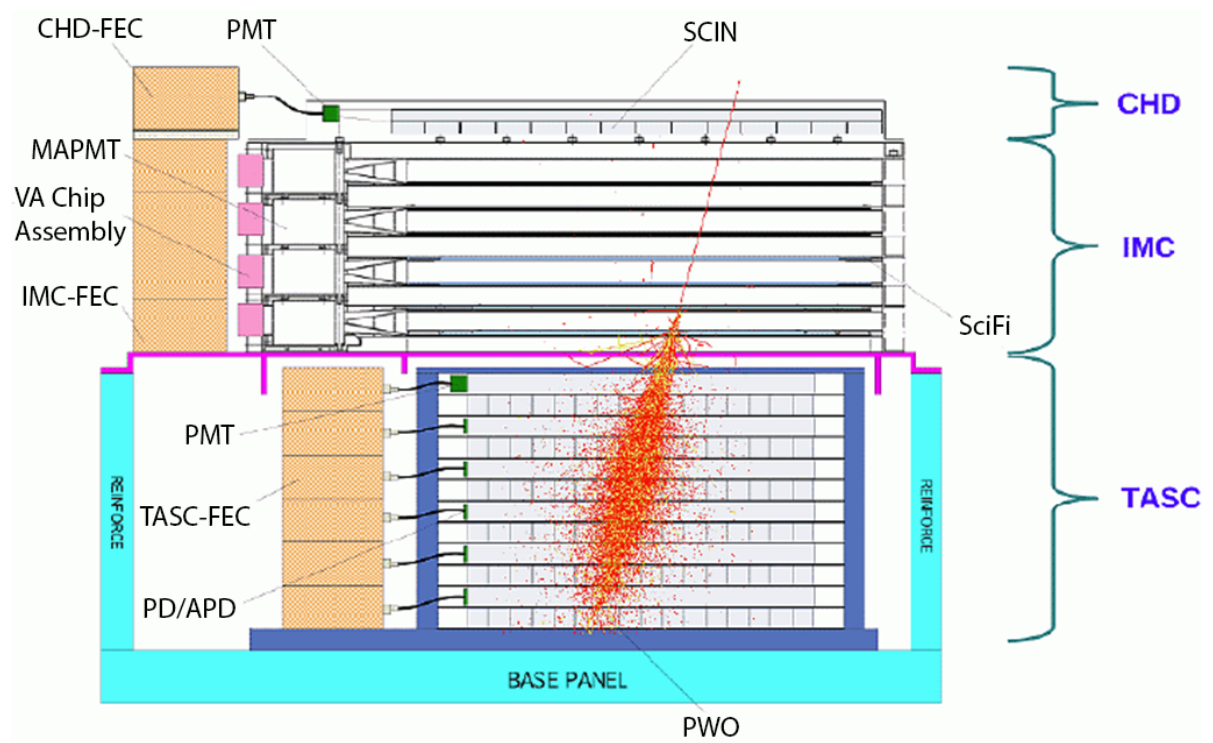

Figure 1: a schematic side view of the CALET instrument.

segmented, plastic scintillator array. Each scintillator is $32 \mathrm{~mm}$ wide, $450 \mathrm{~mm}$ long and $10 \mathrm{~mm}$ thick. The CHD and related front-end electronics are designed to provide incident particle identification over a large dynamic range for charges from $\mathrm{Z}=1$ to $\mathrm{Z}=40$ with sufficient charge resolution $[2,3]$ to resolve individual elements ( $\simeq 0.15 e$ for light elements and $\simeq 0.3 e$ in the Fe region and above).

The IMC images the early shower profile with a fine granularity by using $1 \mathrm{~mm}^{2}$ scintillating fibers individually readout by multi-anode photomultipliers (MAPMT). It consists of 7 layers of tungsten plates, each separated by 2 layers of scintillating fibers ( $\mathrm{SciFi}$ ) with square cross section, arranged in belts along the $\mathrm{x}$ and $\mathrm{y}$ direction and is capped by an additional x,y SciFi layer pair. The transverse dimensions of the IMC are approximately $45 \mathrm{~cm}$ by $45 \mathrm{~cm}$. The total thickness of the IMC is equivalent to $3 \mathrm{X}_{0}$. The first 5 tungsten-SciFi layers sample the shower every 0.2 $\mathrm{X}_{0}$ while the following 2 layers provide $1.0 \mathrm{X}_{0}$ sampling. The IMC is designed to: (i) separate the incident particles; (ii) determine the starting point of the shower; (iii) reconstruct the incident particle trajectory, and (iv) provide a coarse measurement of the particle's charge. The readout of the SciFi layers consists of multianode photomultiplier tubes with 64 anodes.

The TASC measures the total energy of the incident particle and discriminates electrons and gamma-rays from hadrons. The calorimeter is composed of 12 layers of Lead Tungstate (PWO) "logs", each with dimensions $20 \mathrm{~mm}(\mathrm{H}) \times 19 \mathrm{~mm}(\mathrm{~W}) \times 326 \mathrm{~mm}(\mathrm{~L})$. Alternate layers are oriented perpendicular to each other to provide $\mathrm{x}, \mathrm{y}$ coordinates of the shower core. The total area of the TASC is about $1,024 \mathrm{~cm}^{2}$ and the total thickness is about $27 \mathrm{X}_{0}$ at normal incidence. A protonrejection power better than $10^{5}$ is necessary for observing electrons up to $10 \mathrm{TeV}$. This goal is achieved with the combined information from IMC and TASC. On the other hand, the largest signal (in MIP units) in one log is approximately $10^{5}$ for electrons of $10 \mathrm{TeV}$ and $10^{6}$ MIPs for protons of $1000 \mathrm{TeV}$. Therefore, the readout system for the TASC should have a dynamic range from 0.5 to $10^{6}$ MIPs [4]. To measure the energy deposit in this range with six orders of magnitude, a Silicon avalanche photodiode (APD) is used in addition to a Si PIN-photodiode (PD). While the top layer 
of the TASC is read out by PMTs, to provide a high energy trigger, layers from the second to the last of PWO are read out from one side with a dual APD/PD package. With this configuration the expected dynamic range is from about 0.5 MIP to about $10^{6}$ MIPs. Charged particles and gamma rays $>10 \mathrm{GeV}$ will be triggered above a $15 \mathrm{MIP}$ threshold from the sum of the last dynodes of the MAPMTs of the last layer of the IMC and a 55 MIP threshold from the sum of the PMTs of the first layer of the TASC. Electrons in the $1 \mathrm{GeV}-10 \mathrm{GeV}$ range will be observed only for a limited exposure by reducing the IMC trigger threshold.

\section{Energy calibration of TASC}

In September 2012 a beam test was carried out at CERN (SPS H8 and H2 lines) using a CALET engineering model to check the calibration method and the performance of the instrument with electrons. The energy calibration of the TASC was performed using Minimum Ionizing Particles (MIPs). The TASC was exposed to a beam of $150 \mathrm{GeV}$ muons at normal incidence on the calorimeter surface [5].

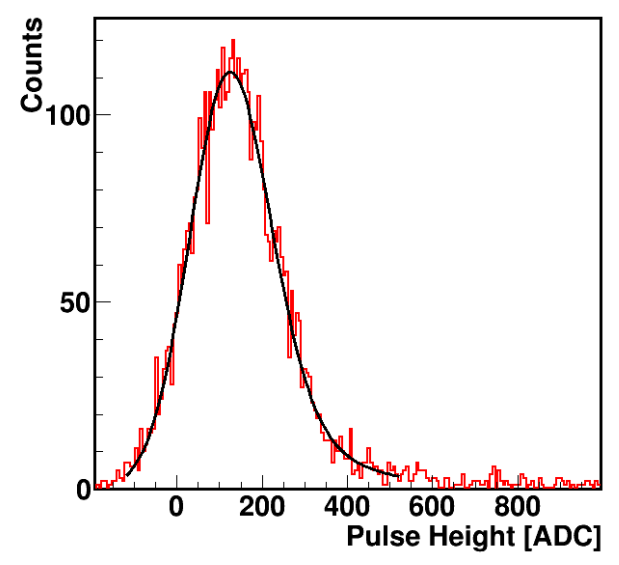

Figure 2: Distribution of PWO signals from a $150 \mathrm{GeV}$ muon beam; the black curve is the result of the fit to a Landau distribution convoluted with a Gaussian.
The mean pedestal value of every TASC channel was calculated and subtracted and pulse height spectra were plotted. In order remove unwanted background contributions from nearby crystals, we selected only events in which the MIP trajectory crosses the PWO log of interest. We used the IMC tracking power to select particles crossing each PWO crystal (an example for layer \#2 is given in fig.3(a)). A typical distribution of the deposited energy by a $150 \mathrm{GeV}$ muon is shown in figure 2 and fitted to a Landau distribution convoluted with a Gaussian and shown (black line) in the same picture. From the fit procedure we derived the most probable value (MPV) of the energy deposit in each channel.

Normalizing each PWO $\log$ to the signal value corresponding to the MPV, all the detector channels were equalized and calibrated. In order to verify the TASC response with electrons it was necessary to assign for each channel the appropriate readout scale by choosing between the HIGH and LOW APD signals on an event by event basis.

The correlation between HIGH and LOW APD range signals, for one TASC log, is shown in figure 3(b); it can be noticed that the HIGH range saturates for signals above $\sim 64 \times 10^{3}$ ADC. HIGH and LOW scales were then inter-calibrated, i.e. for each APD sensor a High gain vs Low gain plot was built and fit to a breakline function. This was used to rescale the low-range signal whenever the corresponding high-range signal started deviating from linearity. 


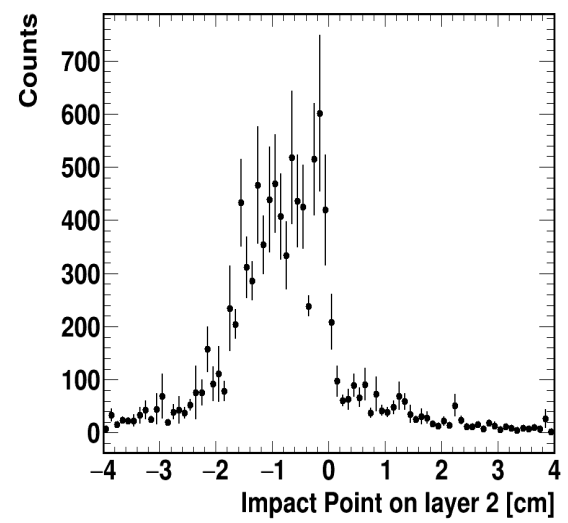

(a)

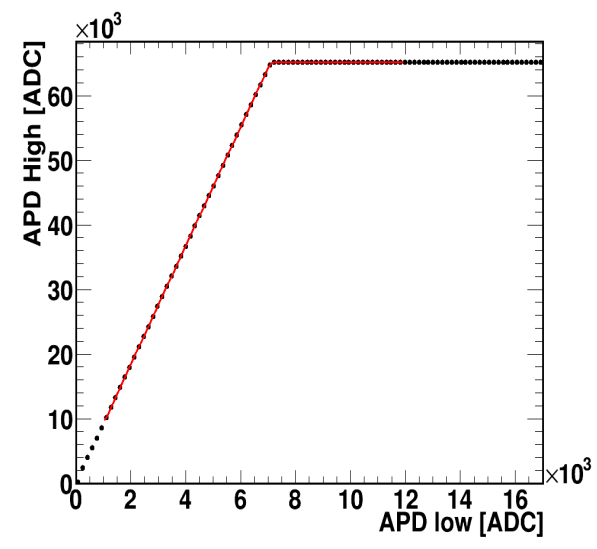

(b)

Figure 3: (a) a PWO log signal as a function of particle impact point in a direction transverse to the beam; (b) correlation plot between APD High gain and Low gain.

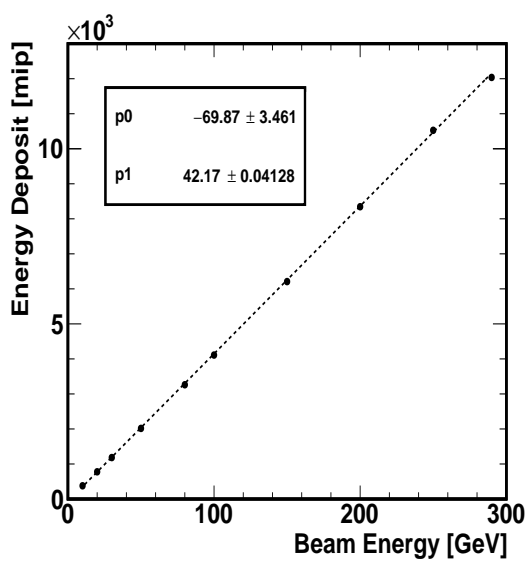

(a)

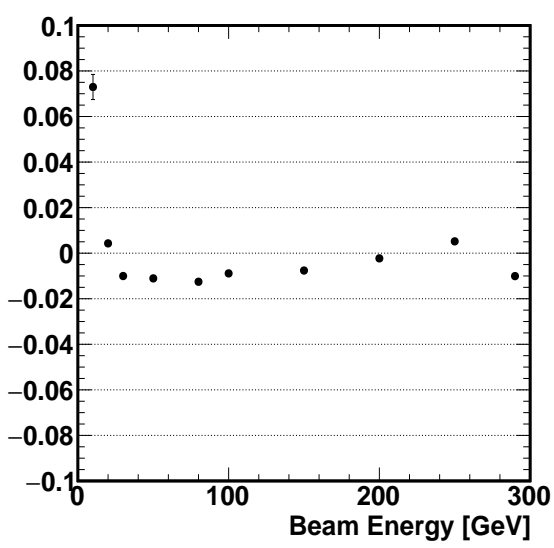

(b)

Figure 4: (a) linearity curve of the TASC with electrons; (b) residuals versus nominal beam energy.

\section{TASC response to electrons}

The TASC calorimeter was tested with electron beams at different energies $(10,20,30,50,80$, $100,150,200,250$ and $290 \mathrm{GeV}$ ) impinging in the middle of its upper surface. The total deposited energy was calculated, event by event, by summing the signals of all logs above a threshold equal to $20 \%$ of 1 MIP signal to cut off the electronic noise contribution. In figure 4(a) the mean energy deposit in the TASC is shown as a function of the nominal beam energy. The experimental data were fitted to a linear function. In figure 4(b) the residuals between data and the linear curve from the fit are shown. With the exception of $10 \mathrm{GeV}$ electrons, a good linearity within $\pm 2 \%$ is achieved 
up to $290 \mathrm{GeV}$.

The energy resolution of the TASC e.m. showers is shown in figure 5. Being a homogeneous calorimeter, the energy resolution is determined by fluctuations in the signals (light yield, photoelectron statistics), by fluctuations on the shower (starting point, development and leakage) and by instrumental effects (noise, light attenuation, non-uniformity). The electron energy resolution was fitted to a function given by the sum in quadrature of three terms:

$$
P_{0} \oplus \frac{P_{1}}{\sqrt{E[G e V]}} \oplus \frac{P_{2}}{E[G e V]}
$$

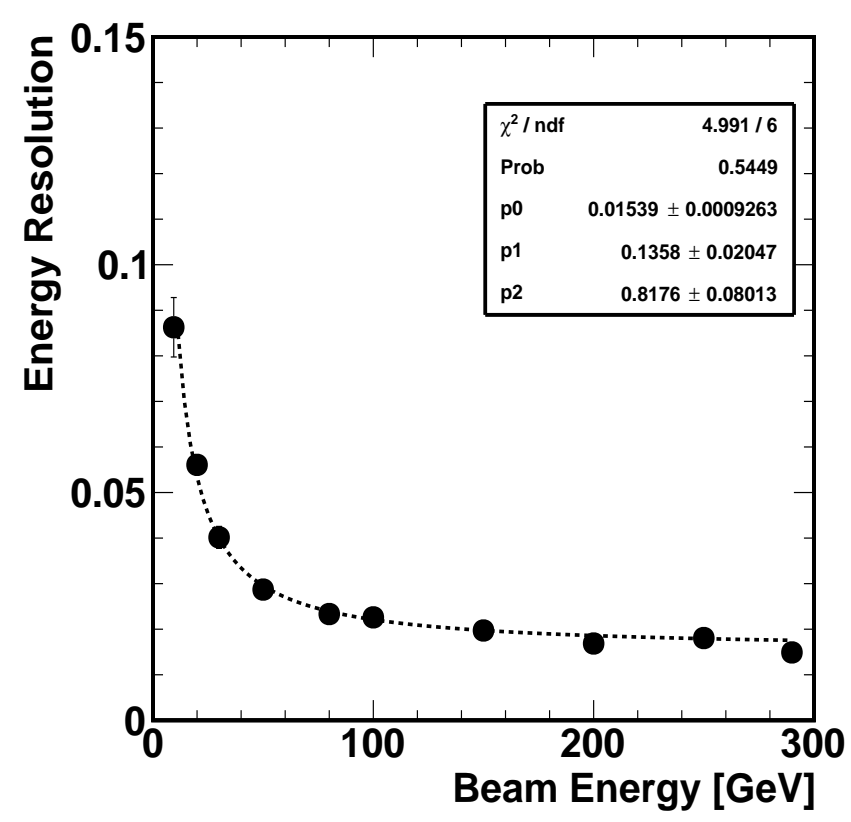

Figure 5: energy resolution for electrons from 10 to $290 \mathrm{GeV}$.

where the $P_{0}$-term (constant) represents the contributions due to finite size of calorimeter and to non-uniformities of the detector, the $P_{1}$-term represents the photo-statistics contribution $\left(\sim \frac{1}{\sqrt{E}}\right)$ and the $P_{2}$-term represents the electronic noise contribution $\left(\sim \frac{1}{E}\right)$. From the fit we obtained $P_{2}=(81 \pm 8) \%, P_{1}=(13 \pm 2) \%$ and $P_{0}=(1.5 \pm 0.1) \%$. The high value of the $P_{2}$-term id probably due to the low signal to noise ratio $(\sim 1)$ for MIP energy deposits from the APD sensors mounted on the prototype used for the test.

\section{Summary}

The calorimeter apparatus of CALET was successfully tested at CERN with electrons confirming the expected performances from MonteCarlo simulations. The TASC prototype showed a 
good resolution in energy with an asymptotic value of $\sim 1.5 \%$. The analysis results demonstrates that the calibration method, based on MIP signals (in this case with muons), is well undestood and it can be adopt on orbit for CALET flight model. The CALET mission is scheduled to be launched on August $16^{\text {th }}$ from the Tanegashima Space Center (Japan).

\section{References}

[1] K.Yamaoka et al.: Proc. of the 32nd Int.Cosmic Ray Conference (2011)

[2] Y. Shimizu et al.: Proc. of the 32nd Int.Cosmic Ray Conference (2011)

[3] P.S.Marrocchesi et al.: Nucl. Instrum. Meth. A 659477 (2011)

[4] I.Daijito et al.: Proceedings of the $32^{\text {th }}$ ICRC (2011)

[5] T.Tamura et al.: Proceedings of the $33^{\text {th }}$ ICRC (2013) 